Int. J. Contemp. Math. Sciences, Vol. 2, 2007, no. 32, 1601 - 1606

\title{
Nuclearity and Multipliers between Banach Spaces
}

\author{
Erdal Karapinar \\ Atılım University, Department of Mathematics \\ Kızılcaşar Mahallesi, 06836 İncek Gölbaşı, Ankara, Turkey \\ ekarapinar@atilim.edu.tr
}

\begin{abstract}
Let $\ell$ be a Banach sequence space with a monotone norm $\|\cdot\|_{\ell}$, in which the canonical system $\left(e_{i}\right)$ is a normalized symmetric basis. Let $\Lambda$ be the class of such $\ell$ spaces and $\mathcal{D}(\ell, \tilde{\ell})$ be the space of all diagonal operators (that is multipliers) acting between $\ell, \tilde{\ell} \in \Lambda$. In [2], Djakov and Ramanujan considered the special case of multipliers on the class of Orlicz sequence spaces and proved that for Orlicz functions, if $\ell=l_{M}$ and $\tilde{\ell}=l_{N}$, then $\mathcal{D}\left(l_{M}, l_{N}\right)=l_{M_{N}^{*}}$, where $l_{M_{N}^{*}}:=\sup \{N(s t)-M(t)$ : $t \in(0,1)\}$.

We consider the general form of multipliers on the class $\Lambda$ and evaluate for some well known Banach sequence spaces. In Theorem 2.7 , it is observed that quasidiagonal isomorphisms of different $\ell$-Köthe spaces implies nuclearity which coincide with the common multipliers $(\Delta(\ell, \tilde{\ell}):=\mathcal{D}(\ell, \tilde{\ell}) \cap \mathcal{D}(\tilde{\ell}, \ell))$ of the corresponding spaces $\ell \in \Lambda$ and some results of [1] and [7] become the consequence of this theorem.
\end{abstract}

Mathematics Subject Classification: 46A45, 47A99

Keywords: Nuclearity, $\ell$-Köthe Spaces

\section{Introduction}

Let $\ell$ be a Banach sequence space in which $\left\{e_{i}=\left(\delta_{i, j}\right)_{j \in \mathbb{N}}: i \in \mathbb{N}\right\}$ forms an unconditional basis. The norm $\|\cdot\|_{\ell}$ is called monotone [3] if $\|x\|_{\ell} \leq\|y\|_{\ell}$ whenever $x=\left(\xi_{i}\right), y=\left(\eta_{i}\right),\left|\xi_{i}\right| \leq\left|\eta_{i}\right|, i \in \mathbb{N}$. By $\Lambda$ we denote the set of all such spaces $\ell$ with monotone norm and by $\Lambda^{(s)}$ the class of those of them with symmetric canonical basis $\left\{e_{i}\right\}$.

A matrix $A:=\left(a_{i, n}\right)_{i, n \in \mathbb{N}}$ of real numbers is called a Köthe matrix if $0 \leq$ $a_{i, n} \leq a_{i, n+1}$ for each $i, n \in \mathbb{N}$; and for each $i \in \mathbb{N}$, there is $n \in \mathbb{N}$ such that $a_{i, n}>0$. 
Definition 1.1. For $\ell \in \Lambda$ the $\ell$-Köthe space $K^{\ell}(A)$ (see $[5,6,8]$ ), defined by the Köthe matrix $A=\left(a_{i, n}\right)_{i, n \in \mathbb{N}}$, is a Fréchet space of number sequences $\xi=\left(\xi_{i}\right)$ such that $\left(\xi_{i} a_{i, n}\right) \in \ell$, for each $n$, with the topology generated by the system of seminorms $\left\{\left|\left(\xi_{i}\right)\right|_{n}:=\left\|\left(\xi_{i} a_{i, n}\right)\right\|: n \in \mathbb{N}\right\}$.

Note that $\left|\left(e_{i}\right)\right|_{n}=\left|\left(e_{i} a_{i, n}\right)\right|=a_{i, n}$. Hereafter the notation $e=\left(e_{i}\right)_{i \in \mathbb{N}}, e_{i}:=$ $\left(\delta_{i, k}\right)_{k \in \mathbb{N}}$, will be always used for the canonical basis of $K^{\ell}(A)$ regardless of a matrix $A$. When $\ell$ is an $l_{p}$, we obtain the usual Köthe space

$K^{l_{p}}(A)=\left\{(\xi)=\left(\xi_{i}\right):\left|\left(\xi_{i}\right)\right|_{n}^{p}=\sum_{i=1}^{\infty}\left|\xi_{i}\right|^{p}\left(a_{i, n}\right)^{p}<\infty, \forall n \in \mathbb{N}\right\}$. Due to [11], it is known that every Fréchet space with an absolute basis is isomorphic to some $l_{1}$-Köthe space.

Set $\mathcal{P}:=\left\{a=\left(a_{i}\right)_{i \in \mathbb{N}}: a_{i} \geq 1, \forall i\right\}$. For $a \in \mathcal{P}$ we introduce the weighted $\ell$-space as $\ell(a):=\left\{x=\left(\xi_{i}\right):\|x\|_{\ell(a)}:=\left\|\left(\xi_{i} a_{i}\right)\right\|_{\ell}<\infty\right\}$. For a given sequences $a=\left(a_{i}\right)_{i \in \mathbb{N}} \in \mathcal{P}$ and $\lambda_{n} \rightarrow \alpha,-\infty<\alpha \leq \infty$, we call the $\ell$-Köthe space $E_{\alpha}^{\ell}(a):=K^{\ell}\left(\exp \left(\lambda_{n} a_{i}\right)\right), \ell$-power series space of finite (respectively, infinite) type if $\alpha<\infty$ (respectively, $\alpha=\infty$ ).

Let $X=K^{\ell}(A)$ and $\tilde{X}=K^{\ell}(\tilde{A})$ be $\ell$-Köthe spaces with the canonical bases $\left(e_{i}\right)$. We say that $X$ is quasidiagonally isomorphic to $\tilde{X}$ and write $X \stackrel{q d}{\simeq} \tilde{X}$ if there exists $T: X \rightarrow \tilde{X}$ such that $T e_{i}:=t_{i} e_{\varphi(i)}, \quad i \in \mathbb{N}$, is an isomorphism, where $t_{i}$ is a sequence of numbers and $\varphi: \mathbb{N} \rightarrow \mathbb{N}$ is a bijection.

A function $M:[0, \infty) \rightarrow[0, \infty)$ is said to be an Orlicz function if it is convex and $M(0)=0 ; \quad \lim _{t \rightarrow \infty} M(t)=\infty$. A function $M$ is said to be degenerate, if it vanishes for some $t>0$. It is clear that any Orlicz function is continuous, non-decreasing and satisfies the condition :CM(t) $\leq M(C t), C>1$.

Each Orlicz function $M$ determines the Banach space $l_{M}$, so called Orlicz space of all scalar sequences $x=\left(\xi_{k}\right)$ such that $\sum_{k=1}^{\infty} M\left(\left|\xi_{k}\right| / r\right)<\infty$ for some $r>0$, equipped with the norm: $\|x\|=\|x\|_{l_{M}}:=\inf \left\{r>0: \sum_{k=1}^{\infty} M\left(\left|\xi_{k}\right| / r\right) \leq\right.$ $1\}$.

We introduce an order relation on the class of all Orlicz functions by setting $M_{1} \preceq M_{2}$ if there are constants $C>0, \delta>0$ such that $M_{1}(t) \leq M_{2}(C t)$ for $t \in[0, \delta]$.

Remark: The relation $\preceq$ is a proper partial order. Since for any Orlicz function $M$ there exists an Orlicz function $N$ which is not comparable with $M$ (see, e.g. [9]). We refer to [10] for the basic theory of Orlicz functions and Orlicz sequence space.

In [1], it is proved that, for different Köthe matrices $A$ and $B$, quasidiagonal isomorphisms of $l_{p}$-Köthe space and $l_{q}$-Köthe space $(1 \leq p \neq q<\infty)$ implies "nuclearity" of the spaces (see 2.4). In [7], quasidiagonal isomorphisms of $l_{M}$-Köthe spaces and $l_{N}$-Köthe spaces for different Orlicz functions $M$ and $N$ implies some kind of the "nuclearity" of the spaces (see 2.5). These two result are generalized in Theorem 2.7, and it is observed that quasidiagonal 
isomorphisms of different $\ell$-Köthe spaces implies nuclearity which coincide with the multipliers of the corresponding spaces $\ell \in \Lambda$ and the results in [1] and [7] become the consequence of this theorem.

\section{Multipliers}

Let $\mathcal{D}(\ell, \tilde{\ell})$ be the space of all diagonal operators (that is multipliers) acting between $\ell, \tilde{\ell} \in \Lambda$. Set $\Delta(\ell, \tilde{\ell}):=\mathcal{D}(\ell, \tilde{\ell}) \cap \mathcal{D}(\tilde{\ell}, \ell)$. One can easily observe the following equalities :

(A) For any $p, q \geq 1, \mathcal{D}\left(l_{p}, l_{q}\right)= \begin{cases}l_{r} \quad \frac{1}{r}=\frac{1}{q}-\frac{1}{p} & \text { if } p>q, \\ l_{\infty} & \text { if } p \leq q,\end{cases}$

(B) $\mathcal{D}\left(l_{1}, \ell\right)=l_{\infty}$ for each $\ell \in \Lambda$,

(C) $\mathcal{D}\left(l_{\infty}, \ell\right)=\ell$ for each $\ell \in \Lambda$,

(D) $\mathcal{D}\left(\ell, l_{\infty}\right)=l_{\infty}$ for each $\ell \in \Lambda$,

(E) $\mathcal{D}\left(\ell, l_{1}\right)=\ell^{*}$ for each $\ell \in \Lambda$, where $\ell^{*}$ is dual of $\ell \in \Lambda$.

$(F) \mathcal{D}(\ell, \ell)=l_{\infty}$ for each $\ell \in \Lambda$; in particular $\mathcal{D}\left(l_{\infty}, l_{\infty}\right)=l_{\infty}$,

(G) $\mathcal{D}(\ell, \tilde{\ell}) \in \Lambda$,

(H) $\mathcal{D}\left(l_{M}, l_{N}\right)=l_{\infty}$ if and only if $M \preceq N$

(I) $\mathcal{D}(\tilde{\ell}, \ell)=\mathcal{D}(\ell, \tilde{\ell})=l_{\infty}$ then $\tilde{\ell}=\ell$.

$(J) \ell \in \Lambda^{(s)}$ if and only if $\mathcal{D}\left(\ell, \ell_{\sigma}\right)=l_{\infty}$ for each bijection $\sigma: \mathbb{N} \rightarrow \mathbb{N}$

It is convenient to realize a Cartesian product of $\ell \in \Lambda$ and $\tilde{\ell} \in \Lambda$, as a Banach sequence space $\underline{\ell \times \ell}$ such that $(x, y) \rightarrow z=\left(\zeta_{i}\right)$ and $\zeta_{i}=$ $\left\{\begin{array}{cc}\xi_{k} & \text { if } \quad i=2 k-1, \\ \eta_{k} & \text { if } \quad i=2 k,\end{array}\right.$ where $x=\left(\xi_{k}\right) \in \ell$ and $y=\left(\eta_{k}\right) \in \tilde{\ell}$.

For $1 \leq p<q<\infty$, we easily obtain that

1. $\mathcal{D}\left(\underline{l_{q} \times l_{p}}, \underline{l_{p} \times l_{q}}\right)=\underline{l_{\infty} \times l_{r}}$,

2. $\mathcal{D}\left(\underline{l_{p} \times l_{q}}, \underline{l_{q} \times l_{p}}\right)=\underline{l_{r} \times l_{\infty}}$, where $\frac{1}{r}=\frac{1}{q}-\frac{1}{p}$.

Theorem 2.1. For $\ell, \tilde{\ell} \in \Lambda$ the following are equivalent:

(i) $\ell \neq \tilde{\ell}$

(ii) One of the $\mathcal{D}(\tilde{\ell}, \ell), \mathcal{D}(\ell, \tilde{\ell})$ is non-trivial (that is, not equal to $l_{\infty}$.) 
Proof. Regarding $(F)$ and $(I), \ell=\tilde{\ell}$ if and only if $\mathcal{D}(\ell, \tilde{\ell})=l_{\infty}=\mathcal{D}(\tilde{\ell}, \ell)$.

Theorem 2.2. If $\ell \neq \tilde{\ell}$ then

1. there exists a Köthe matrix $A$ such that $K^{\ell}(A) \neq K^{\tilde{\ell}}(A)$

2. there exists a positive sequence $a=\left(a_{i}\right)$ such that $E_{\nu}^{\ell}(a) \neq E_{\nu}^{\tilde{\ell}}(a), \nu=$ $0, \infty$.

Proof. We consider the case $(i)$, since the case $(i i)$ can be obtained analogously. If $\ell \neq \tilde{\ell}$ then by the Theorem 2.1 , one of the $\mathcal{D}(\tilde{\ell}, \ell), \mathcal{D}(\ell, \tilde{\ell})$ is non-trivial. Without loss of generality, say $\mathcal{D}(\ell, \tilde{\ell}) \neq l_{\infty}$. Thus for each $x=\left(\xi_{i}\right) \in \ell$ there exist $\lambda=\left(\lambda_{i}\right) \in \mathcal{D}(\ell, \tilde{\ell})$, such that $\left(\lambda_{i} \xi_{i}\right) \in \tilde{\ell}$.

Suppose $\left(a_{i p}\right):=\left(\lambda_{i}\right)$ for each $p$. Thus, $\left(\xi_{i} a_{i p}\right) \in \ell$ and also $\left(\xi_{i} a_{i p}\right) \in \tilde{\ell}$ which implies that $K^{\ell}(A) \subset K^{\tilde{\ell}}(A)$. But $K^{\ell}(A) \not \supset K^{\tilde{\ell}}(A)$. Indeed, if not, $\left(\xi_{i}\right) \in K^{\tilde{\ell}}(A)$ and $\left(\xi_{i} a_{i p}\right) \in \tilde{\ell}$ which implies that $\left(\xi_{i} a_{i p}\right) \in \ell$. Thus, $\left(a_{i p}\right) \in$ $\mathcal{D}(\tilde{\ell}, \ell)$ which contradicts the assumption $\mathcal{D}(\tilde{\ell}, \ell)=l_{\infty}$.

Nuclearity of the classical Köthe spaces $K^{l_{p}}(A)$ is equivalent to the GrothendieckPietsch condition [13]; $\forall p \quad \exists q>p$ and $\left(\xi_{n}\right) \in l_{1}$ with $a_{n}^{p} \leq \xi_{n} a_{n}^{q}, \quad n \in N$. In [14], it is proved that $K^{\ell}(A)$ is nuclear if and only if $A$ satisfies the GrothendieckPietsch condition.

Lemma 2.3. $K^{l_{p}}\left(a_{n k}\right)$ is nuclear if and only if

$$
\exists r \forall k \exists \sigma(k): \sum_{i=1}^{\infty}\left(\frac{a_{n k}}{a_{n \sigma(k)}}\right)^{r}<\infty
$$

where $\sigma: \mathbb{N}: \rightarrow \mathbb{N}$ is an injection map.

Proof. It is well known fact that (see [12]) $K^{p}\left(a_{n k}\right)$ is nuclear if and only if

$$
\sum_{i=1}^{\infty} \frac{a_{n k}}{a_{n \phi(k)}}<\infty
$$

So it is sufficient to show that (2.3) implies (2). Assume that (2.3) holds. Fix $r \in \mathbb{N}$, for any $k \in \mathbb{N}$ there exists an injective map $\phi: \mathbb{N} \rightarrow \mathbb{N}$ such that $\sum_{i=1}^{\infty}\left(\frac{a_{n k}}{a_{n \phi(k)}}\right)^{r}<\infty$, that is, $\left(\frac{a_{n k}}{a_{n \phi(k)}}\right) \in l_{r}$. In the same way, for $\phi(k)$, there exist $\phi^{2}(k)$ such that $\sum_{i=1}^{\infty}\left(\frac{a_{n \phi(k)}}{a_{n \phi^{2}(k)}}\right)^{r}<\infty$, that is, $\left(\frac{a_{n \phi}(k)}{a_{n \phi^{2}(k)}}\right) \in l_{r}$.

If we determine recursively $\phi^{s}(k)$, for $s=1, \ldots, r$ so that $\sum_{i=1}^{\infty}\left(\frac{a_{n \phi^{s-1}(k)}}{a_{n \phi^{s}(k)}}\right)^{r}<\infty$, that is, $\left(\frac{a_{n \phi^{s-1}(k)}}{a_{n \phi^{s}(k)}}\right) \in l_{r}$. 
So we have, $\left(\frac{a_{n k}}{a_{n \phi^{r}(k)}}\right)=\left(\frac{a_{n k}}{a_{n \phi(k)}}\right) \cdot\left(\frac{a_{n \phi}(k)}{a_{n \phi^{2}(k)}}\right) \ldots\left(\frac{a_{n \phi^{r}-1}(k)}{a_{n \phi^{r}(k)}}\right)$

By generalized Hölder's inequality

$$
\begin{gathered}
\sum_{n=1}^{\infty}\left|\left(\frac{a_{n k}}{a_{n \phi^{r}(k)}}\right)\right|=\sum_{n=1}^{\infty}\left|\left(\frac{a_{n k}}{a_{n \phi(k)}}\right) \cdot\left(\frac{a_{n \phi}(k)}{a_{n \phi^{2}(k)}}\right) \ldots\left(\frac{a_{n \phi^{r-1}(k)}}{a_{n \phi^{r}(k)}}\right)\right| \\
\leq C\left\|\left(\frac{a_{n k}}{a_{n \phi(k)}}\right)\right\|\left\|_{l_{r}} \cdot\right\|\left(\frac{a_{n \phi}(k)}{a_{n \phi^{2}(k)}}\right)\|\|_{l_{r}} \ldots\left\|\left(\frac{a_{n \phi^{r-1}(k)}}{a_{n \phi^{r}(k)}}\right)\right\|_{l_{r}}
\end{gathered}
$$

Since all factors are finite, product is finite.

Definition 2.4. $A \in \mathbb{N}(\ell, \tilde{\ell})$ if for each $p$, there exists $q$ such that $\left(\frac{a_{i p}}{a_{i q}}\right) \in$ $\Delta(\ell, \tilde{\ell})$.

Example 2.5. (see[1], Prop.4) If $K^{l_{p}}(A) \stackrel{q d}{\simeq} K^{l_{q}}(B)$ then $K^{l_{p}}(A)$ is nuclear. Indeed $\Delta\left(l_{p}, l_{q}\right)=l_{r}$ and regarding the Lemma 2.3, $A \in \mathbb{N}\left(l_{p}, l_{q}\right)$

Example 2.6. (see[r]) If $K^{l_{M}}(A) \stackrel{q d}{\simeq} K^{l_{N}}(B)$ then $K^{l_{M}}(A)$ is nuclear. Indeed, $\Delta\left(l_{M}, l_{N}\right)=l_{M_{N}^{*}}$ and $A \in \mathbb{N}\left(l_{M}, l_{N}\right)$

Theorem 2.7. For $\ell, \tilde{\ell} \in \Lambda$, if $K^{\ell}(A)=K^{\tilde{\ell}}(A)$, then $A \in \mathbb{N}(\ell, \tilde{\ell})$.

Proof. Let $a_{p}:=a_{i p}$. For each $p$, there exists $q$ such that

$$
\tilde{\ell}\left(a_{p}\right) \subset \ell\left(a_{q}\right) \text { and } \ell\left(a_{p}\right) \subset \tilde{\ell}\left(a_{q}\right)
$$

Take $x=\left(\xi_{i}\right) \in \ell$. Then $\left(\frac{\xi_{i}}{a_{i q}}\right) \in \ell\left(a_{q}\right)$. Due to 3 we have $\left(\frac{\xi_{i}}{a_{i q}}\right) \in \tilde{\ell}\left(a_{p}\right)$ which implies that $\left(\xi_{i} \frac{a_{i p}}{a_{i q}}\right) \in \tilde{\ell}$. Thus $\left(\frac{a_{i p}}{a_{i q}}\right) \in \mathcal{D}(\ell, \tilde{\ell})$. Analogously, we obtain that $\left(\frac{a_{i p}}{a_{i q}}\right) \in \mathcal{D}(\tilde{\ell}, \ell)$ which implies that $\left(\frac{a_{i p}}{a_{i q}}\right) \in \Delta(\ell, \tilde{\ell})$.

Corollary 2.8. $K^{\ell}(A)=K^{\ell_{\sigma}}(A)$ if and only if $\mathbb{N}\left(\ell, \ell_{\sigma}\right)$ where $\sigma: \mathbb{N} \rightarrow \mathbb{N}$ is injection.

For $A=\left(a_{i p}\right)$, if for each $p$, there exist $q, C>0$ and $\sigma: \mathbb{N} \rightarrow \mathbb{N}$ is injection such that $a_{i p} \leq C a_{\sigma(i) q}$ and $a_{\sigma(i) p} \leq C a_{i q}$ then we say that the matrix $\left(a_{i p}\right)$ is $\sigma$-equivalent and denote $\left(a_{i p}\right) \equiv a_{\sigma(i) p}$. Let $\mathcal{G}(A):=\{\sigma: \mathbb{N} \rightarrow \mathbb{N}$ injection : $\left.\left(a_{i p}\right) \equiv a_{\sigma(i) p}\right\}$

Theorem 2.9. $K^{\ell}(A) \stackrel{q d}{\simeq} K^{\ell_{\sigma}}(A)$ if and only if there exists $\gamma \in \mathcal{G}(A)$ such that $A \in \mathbb{N}\left(\ell, \ell_{\sigma \circ \gamma}\right)$

Note that $K^{\ell}(A) \stackrel{q d}{\simeq} K^{\ell_{\sigma}}(A)$ if and only if $K^{\ell}(A) \stackrel{q d}{\simeq} K^{\ell_{\sigma \circ \gamma}}(A)$

Problem 2.10. For Tsirelson space $\mathbb{T}$ (see [4]), we observe $\mathcal{D}\left(l_{2}, \mathbb{T}\right)=l_{\infty}$ We claim that $l_{p} \subset \Delta\left(l_{2}, \mathbb{T}\right) \subset l_{\infty}$ and also $\mathcal{D}\left(\mathbb{T}, l_{2}\right) \neq l_{\infty}$ but what exactly it is? 


\section{References}

[1] P. B. Djakov, S. Önal, T. Terzioğlu, M. Yurdakul, Strictly Singular Operators and Isomorphism of Cartesian Products of Power Series Spaces, Arch. Math., 70(1998), 57-65.

[2] P. B. Djakov, M. S. Ramanujan, Multipliers Between Orlicz Sequence Spaces, Turkish J. Math., 24(2000), 313-319.

[3] M. M. Dragilev, Basis in Köthe spaces,(in Russian), Rostov State University, Rostov-on-Don $(1983,2003)$.

[4] W. B. Johnson, J. Lindenstrauss, Handbook of the Geometry of Banach Spaces Volume 1-2 North-Holland, (2001).

[5] E. Karapınar, On $\ell$-Köthe Spaces. International Journal of Pure and Applied Mathematics, 38 (2)(2007), 223-227.

[6] E. Karapinar, Multirectangular characteristic invariants for power $\ell$ Köthe spaces of first type, J. Math. Anal. Appl., 335 (1)(2007), 79-92, , doi:10.1016/j.jmaa.2007.01.049.

[7] E. Karapinar, V. P. Zahariuta, Quasidiagonal Isomorphism of Cartesian Products of Orlicz Power Series Spaces, (to appear).

[8] E. Karapınar, M. Yurdakul, V. P. Zahariuta, Isomorphisms of Cartesian products of $\ell$-power series spaces, Bull. Polish Acad. Sci. Math., $\mathbf{5 4}(2)(2006), 103-111$.

[9] M. A. Krasnoselskii, Ya. B. Rutickii, Convex Functions and Orlicz Spaces, Noorhoff Ltd., Groningen (1961).

[10] J. Lindenstrauss, L. Tzafriri, Classical Banach Spaces I,II, Springer, Berlin, (1977, 1979).

[11] B. S. Mityagin, Approximative dimension and bases in nuclear spaces, (in Russian), Usp. Mat. Nauk., 16 (1961), 63-132.

[12] D. Vogt, R. Meise, Introduction to Functional Analysis, Springer, Berlin (1997).

[13] A. Pietsch, Nukleare lokalkonvexe Räume . Berlin (1965).

[14] T. Terzioğlu, Diametral Dimension and Köthe Spaces, Turkish J. Math. (to appear).

Received: July 10, 2007 